\title{
Spatial Information Sharing Technology Based on Grid
}

\author{
Hong-bin Zhang ${ }^{1,2,3}$, Bao-rui Chen ${ }^{1,2,3}$, Gang $\mathrm{Li}^{2,3}$, and Xiao-ping Xin ${ }^{1,2,3, *}$ \\ ${ }^{1}$ Hulunber Grassland Ecosystem Observation and Research Station, Beijing 100081, China \\ ${ }^{2}$ Key Laboratory of Resource Remote Sensing and Digital Agriculture, \\ Ministry of Agriculture, Beijing 100081, China \\ ${ }^{3}$ Institute of Agricultural Resources and Regional Planning, \\ Chinese Academy of Agricultural Sciences, Beijing 100081, China \\ xinxp@sina.com
}

\begin{abstract}
This paper analyzes the status of spatial data sharing technology. The grid technology based spatial information sharing platform has been adopted to solve the problem of "Isolated Information Islands". A Grid technology-based information sharing system is proposed and analyzes key technologies and processing mechanism.
\end{abstract}

Keywords: Grid, Information Sharing, metadata, geospatial information.

\section{Introduction}

Sharing of Geospatial information has great positive effect to economy and social development. But the data management systems built by different technique systems can not be accessed by each other, thus a lot of "isolated information islands" come into being. How to integrate these heterogeneous data sources distributed in different sites and to realize management and sharing quickly have become research focus at present.

\section{Present Situation and Analysis of Geospatial Information Sharing}

\subsection{Present Situation of Sharing Geospatial Information}

Under the promotion of application demands based on Geospatial information and with the development of Internet technology, relative research organizations and institutions promote one after another information publishing platforms and sharing systems. The main typical systems are as follows.

TerraServer: is an aerial images and satellite images publishing system designed and developed by Microsoft. Users can browse and download images in designated area by Internet.

${ }^{*}$ Corresponding author. 
TerraShare: is a production of $\mathrm{Z} / \mathrm{I}$ imaging company. It is modularized and client/server mode geography images management and publishing system. It can deal with remote sensing images and altitude modules and digital grid images.

Google Earth: is invented earth software developed by Google Company. It can publish images and vector data based on three-dimensional model and provide popular GIS functions such as inquiry, measurement, path query and so on. The systems of the kind include World Wind, Visual Earth and so on.

The research work in spatial information sharing of our country includes two aspects: Scientific data sharing engineering: Science and Technology Department began to implement scientific data sharing engineering from 2002 and in 2003 started up to build sharing centre of mapping and earth system scientific data and sharing service experimental unit and to provide data query and download service to public.

Chinese Spatial information Network: Chinese Remote Sensing Centre has already taken advantage of advanced information technique fully to built large scale and comprehensive spatial information professional website-"Chinese Spatial Data Network"based on spatial information sharing work and experience in recent years, emphasized to set up data sharing mechanism, focused on sharing spatial information. The network realized spatial information directory query and content query and download orders.

\subsection{Existed Problems}

Compared with advanced countries in the world, our country fall behind in spatial information development, utilization, obtaining, management, updating and services.

(1) Lack of integration of spatial information resources

(2) Short of standardized state spatial information resources

(3) Backward management and low sharing level

(4) Low level of automatic obtaining spatial information

(5) Backward infrastructure of spatial information resources

\subsection{Traditional Geospatial Information Sharing}

At present traditional spatial information sharing methods includes: (1) data format conversion mode(2) data direct access mode (3) data mutual operation mode (4)Web GIS data sharing mode. But the traditional methods can not realize mutual operation between heterogeneous spatial data and different Web GIS platforms.

\subsection{Analysis of Implementation Technology}

Comprehensive analysis of features and techniques in spatial information publishing systems based on Internet, there are two sharing methods. 
(1) Users obtain needed spatial information through spatial meta data query and share them by storing in physical mediums or downloading from internet. This method can be called off-line manual sharing mode. The technique matter is to publish and browse spatial information with the aid of web technique and to provide downloading service through HTTP and FTP agreements, such as TerraServer and Chinese Spatial information Network.

(2) Publishing remote sensing images and providing simple applications of query and browse by special clients or browse plug-ins. The technique essence is that clients adopt special agreements to obtain data from servers. The servers usually adopt HTTP service or web services such as Google Earth.

Analysis above two methods, we can see that sharing methods and their technique means in present systems have two aspects shortages.

\section{(1) Present sharing methods can not eliminate "isolated information islands" truly}

The development of internet technique already can resolve information publishing easily, but can not resolve information sharing well. Storage systems used incompatible access methods however, thus remote sensing resources become isolated to users. With the building of network mapping service systems in different technique structures, the isolated information islands are not only not decreased but also increased.

From the technical means to analyze, that is because an easy sharing environment is wanting in internet based on TCP/IP agreement to resolve sharing issues involved in distributed and heterogeneous system, such as resources management, service publishing and safety problems.

\section{(2) Can not share stored resources}

Spatial information has the characteristic of mass memory. In a variety of application systems providing high speed and stability of the stored data service have a larger proportion of cost. Obviously, browse, download off the line of the way not only can not share storage resources, but also create the great wastage of storage resources in data circulation renew.

From the technical means to analyze, mainly because the technical level has not yet been able to direct support for remote sensing data owners to provide a summary way, that will be able to at a lower cost to wan other users to provide real-time online, stable and reliable data access service. On the other hand, internet will not be able to provide convenient integration service directly for data users. Difficulty lies in how to publish the service of information, how to realize data query, how to integrate services in application systems and how to control the data access and so on.

Emerge of grid technique proved chance to resolve above questions.

\section{Grid Technology}

Grid is the third generation of internet application following by Internet and www. Traditional internet realizes union of computer hardware. Web realizes union of web pages. But grid tries to realize complete union of all resources on the Internet and 
provides weldless and compositive calculation and cooperation environment and provides fictitious and limitless calculation and distributed data resources and realizes resources sharing of fictitious organization and question solution.

Based on the research work and experience of key theories and techniques on grid calculation including resources management, information safety, information services, data management and application development environment, America Argonne national lab developed Globus Toolkit. It is grid calculation tool software which can running in every platform. At the same time, it is a grid application environment and development platform. Globus toolkit of development based on the grid application has provided on several areas of support.

(1) Grid safety. It can assure grid calculation environment to run regularly. Globus mainly combines sophisticated distributed safety technologies and expands to some extent in order to adapt to features of grid calculation environment.

(2) obtain and publish of grid information. How to publish, query and search resources information in grid calculation environment are preconditions for utilization resources effectively.

(3) Grid resources management. Because resources in grid environment mainly located in wan wide area network environment, frequently-used lan local area network resources management technologies can not be used effectively. So Globus realizes higher level resources management. Under support of information services, it can manage resources in wan effectively.

(4) Grid long distance data transmission. To realize high speed and reliable data transmission in wan and transparent long distance file I/O access of applications.

To sum up, the core of grid conception is resources sharing and cooperation work. Grid users can use all resources including calculation, storage and data conveniently, but it is not need to know who provides the services. On demands is the aim that grid tries to realize. Some person sum up the function of grid technology is to provide the most important resources for the most needed persons in the most appropriate time.

Advance and development of grid technology raise the hopes to solve difficulties in spatial information sharing and services fundamentally. Introduction grid technology to spatial information utilization and application has become important task at present for us.

\section{Spatial Information Sharing Service Based on Grid}

\subsection{Technical Line}

Spatial information grid technology is based on basic grid technology. The basic frame and function component of spatial information sharing platform is set up based on the technical frame, resources management, information services, safety architecture provided by basic grid laying the foundation for spatial information grid application system. The spatial information grid system architecture is as follow. 


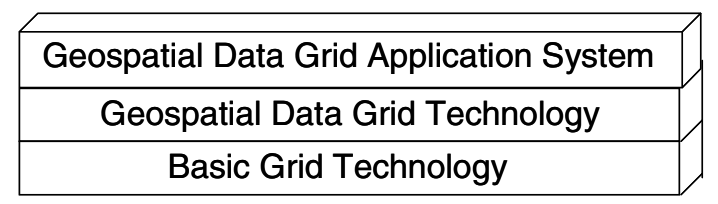

Fig. 1. Spatial information grid

\section{2 Sharing Platform Frame}

Grid middleware is adopted to eliminate isolated information islands. Spatial information sharing platform frame based on grid middleware is designed to realize transparent integration of spatial information resources. The grid middleware is the score to realize sharing spatial information taking the charge of transparent and dynamic access distributed spatial information resources. In the frame, there are several parts.

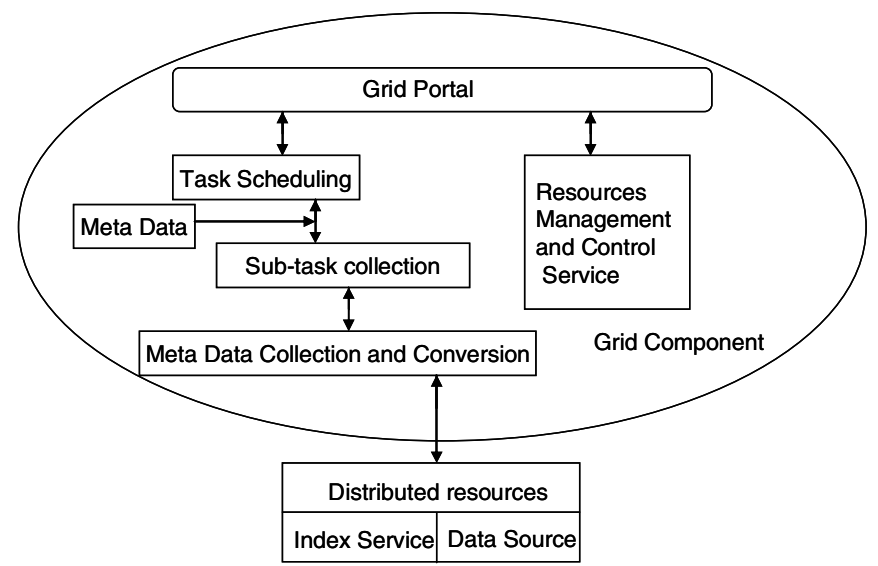

Fig. 2. Grid-based Geospatial sharing frame

(1)Grid data resources. That is rock-bottom distributed spatial information resources which can be accessed by internet. Mainly include two parts: (1) space source, that is the basic unit of information stored and it can be database or distributed heterogeneous spatial data source; (2) information search service, mainly mean through spatial information source to establish an index and to provide index service to upper.

(2)Task scheduling service, this part is mainly responsible for transferring request from spatial information portal, decomposition calls for resources, processing tasks and management of scheduling tasks.

(3)Meta data collection and conversion. According to the results of task scheduling, information collecting task service is localization converted to meet distributed spatial information resources format.

(4)Spatial information resources control service. This part is the control centre of the whole frame and makes every grid component in the reunification of the standard and includes system configuration and index services. 


\subsection{Key Technologies}

(1) Presentation of information and meta data

Presentation of information reflects in two aspects: the first presentation is storing information in database or other storage medium; the second presentation is interface presented to users. Meta data abstracts description of data objection in order that information can be expressed by relationship of elemental attribute and value. We call the mate data as middle layer of data presentation. Generally speaking, data presentation can set up several middle layers and every application program logic layer has corresponding data presentation middle layer. Presentation of meta data can take advantage of semantics web technology fully.

(2) Task scheduling and resources management

Grid resources is complicated, numerous, varied and large. So how to manage all resources in the grid effectively is a painstaking task. Task scheduling mainly take the charge of resolving requirements transferred from spatial information portal, and decomposing of resources scheduling demands and scheduling tasks and management scheduling tasks. Task scheduling can dynamic decompose and schedule tasks according to every site disposition situation in grid in order to improve running efficiency. So scheduling algorithm will affect system efficiency directly.

\section{(3) Safety technique}

The aim of grid is to connect all the information service sites in the internet in order that all users can use information services. Because sites of grid located in different places, how to share data resources safely between sites and how to assure integrity of sharing data are questions to be solved at present. Grid safety authentication mechanism mainly includes identification authentication, information access authentication, audit mechanism. When setting up perfect safety mechanism, it should be avoided that safety authentication will waste too much processing capacity. If system resources used up by safety authentication is more than calculation resources saved by system, grid will lose itself true means. Fault-tolerant and automatic crash recovery should also take into consideration in grid system.

\section{$5 \quad$ Results}

Grid technique is a new generation of internet. Resources sharing is the most important mode in grid technique application. With the aid of grid, combined with spatial information sharing situation, study spatial data sharing and services based on grid in order to perfect spatial information sharing and application environment.

Acknowledgments. This work is supported by National Natural Science Foundation of China (Grant No: 41101383) and the Key Scientific Project of Inner Mongolia (Grant no.20091403) and Scientific Project of Agriculture (Grant no. 201003019, 201003061, 200903060) and Meteorological science and technology of China (Grant No: GYHY200906029-2). 


\section{References}

1. Qiang, W., Jin, H., Shi, X., Zou, D.: A Novel-Based Access Control Model for Grid. In: Jin, H., Pan, Y., Xiao, N., Sun, J. (eds.) GCC 2004. LNCS, vol. 3251, pp. 293-300. Springer, Heidelberg (2004)

2. Cao, H., Spooner, D.P., Jarvis, S.A., Nudd, G.R.: Grid load balancing using intelligent agents. Future Generation Computer System 21(1), 135-149 (2005)

3. OGC 07-006rl. OpenGis catalogue services specification version 2. Open Geospatial Construction (2007)

4. Wang, L.: Geospatial data sharing based on grid. Journal of Computer Application 30, 60-63 (2010)

5. Feng, H., Fan, H., Li, S.: Study on West Mapping Spatial Information sharing and Service Platform based on grid. Journal of Geomatics 35(3), 56-72 (2010) 